\section{Adaptação dos instrumentos de avaliação de eventos adversos para uso em hospitais brasileiros}

\section{Adjustment of adverse events assessment forms for use in Brazilian hospitals}

Walter Mendes ${ }^{1}$

Claudia Travassos ${ }^{2}$

Mônica Martins'

Priscilla Mouta Marques ${ }^{2}$

${ }^{1}$ Escola Nacional de Saúde Pública Sérgio Arouca da Fundação Oswaldo Cruz.

${ }^{2}$ Centro de Informações Científicas e Tecnológicas da Fundação Oswaldo Cruz.

Apoio financeiro: CNPq. - Processo 403647/2004-5.

Agradecimentos: A Daniel Brasil pela programação e aos pesquisadores canadenses Ross Baker, Peter Norton, Virginia Flintoft e Régis Blais.

Correspondência: Walter Mendes, Rua Leopoldo Bulhões, 1480, Manguinhos, Rio de Janeiro, RJ CEP 21041-210.E-mail: wmendes@ensp.fiocruz.br

\section{Resumo}

Objetivo: Adaptar os instrumentos de avaliação e os critérios de rastreamento do Canadian Adverse Events Study (CAES) para a avaliação da ocorrência de eventos adversos (EAs) em hospitais brasileiros. Metodologia: O processo de adaptação dos instrumentos foi realizado em 4 etapas: (I) tradução do inglês para o português dos formulários; (II) painel de especialistas para julgar com base em consenso os critérios de rastreamento e dúvidas na tradução; (III) pré-teste; e (IV) retro-tradução dos formulários. Resultados: A tradução de EA consensuada no painel de especialista foi "lesão ou dano [injury] não intencional que resulta em incapacidade ou disfunção [disability], temporária ou permanente, e/ou prolongamento do tempo de permanência ou morte como conseqüência do cuidado prestado". O painel modificou a composição dos critérios de rastreamento do formulário do CAES. Resultados do pré-teste questionaram a qualidade da avaliação na fase de rastreamento. Uma avaliação realizada por médico da equipe da pesquisa obteve uma freqüência de potenciais EAs na fase de rastreamento semelhante ao valor obtido no CAES. Um novo treinamento dos revisores foi realizado. Conclusões: Os instrumentos de avaliação adaptados do CAES mostraram-se aplicáveis ao contexto de hospitais brasileiros. O método de revisão retrospectiva de prontuários requer, no entanto, um treinamento bem estruturado e avaliação do desempenho dos revisores previamente a sua incorporação como revisor.

Palavras-chave: Eventos adversos. Adaptação de instrumentos. Qualidade na assistência à saúde. Segurança do paciente. Painel de especialistas.

Aprovado pelo Comitê de Ética em Pesquisa da FIOCRUZ, Protocolo: 271/05 
Abstract

Objective: To adapt assessment forms and triggers of the Canadian Adverse Events Study (CAES), aiming at the assessment of Adverse Events (AEs) in Brazilian hospitals. Method: Adjustment followed a four-step process: (I) translation from Portuguese into English; (II) expert' panel to judge triggers in the context of Brazilian hospitals and translation of the assessment form (III) pretest and (IV) back-translation. Results: The expert panel changed the definition of AEs in Portuguese by introducing the terms harm and dysfunction in "unintended harm or injury resulting in death, temporary or permanent disability or dysfunction, and/ or prolonged hospital stay resulting from health care management". The Panel also modified the list of triggers in the CAES form. Pre-test results detected problems in the quality of assessment in the tracking phase. The frequency of potential AEs obtained by a senior physician was similar to the one obtained by CAES. This indicates that information available in medical records was adequate for using retrospective record review methods, but that nurse reviewers needed to be retrained. Conclusions: Adapted CAES tools and triggers are feasible to be used in hospitals in Brazil. Retrospective chart review requires a well structured training process and assessment of reviewers' performance previously to their acceptance as reviewers.

Key Words: Adverse events. Instruments adjustment. Evaluation methodology. Quality in health care. Patient safety. Expert panel.

\section{Introdução}

Evento adverso (EA) é definido como lesão ou dano [injury] não intencional que resultou em incapacidade ou disfunção [disability], temporária ou permanente, e/ ou prolongamento do tempo de permanência ou morte como conseqüência do cuidado prestado. A alta incidência de EA em hospitais vem preocupando estudiosos, profissionais e gestores em saúde. Estudos recentes apontam que cerca de $10 \%$ dos pacientes internados sofrem algum tipo de $\mathrm{EA}^{1}$.

Mundialmente, a discussão da qualidade do cuidado em serviços de saúde voltase cada vez mais para a questão da ocorrência de $\mathrm{EA}^{1-7}$. Entretanto, poucos são os estudos brasileiros sobre o tema e os trabalhos realizados enfocam causas específicas, como EAs provocados por medicamentos ou decorrentes de complicações de métodos terapêuticos cruentos ${ }^{3}$. A partir de uma revisão sistemática da literatura ${ }^{1}$, foi planejado um estudo de avaliação de EAs para ser desenvolvido em hospitais gerais, de ensino, no Brasil. Esse estudo distingue-se dos demais estudos já realizados no Brasil por ter como objetivo avaliar a ocorrência de EA decorrente dos vários processos encontrados no ambiente hospitalar. Tomouse por base outros estudos, realizados nos EUA, Austrália, Nova Zelândia, Reino Unido, França, Dinamarca e Canadá ${ }^{8-16}$, que utilizaram como metodologia a revisão retrospectiva de prontuários,. Um estudo espanhol $^{17}$, realizado posteriormente a esta pesquisa, usou a mesma metodologia. Optou-se por utilizar o CAES como referência para este estudo por ter incorporado os aperfeiçoamentos sugeridos pelos estudos antecedentes. No estudo australiano ${ }^{11}$, os enfoques médicos legal, que pautaram os estudos iniciais ${ }^{8-10}$, foram modificados para uma abordagem voltada para a melhoria de qualidade. Essa abordagem também foi seguida no estudo canadense ${ }^{16}$.

Existem vários métodos de avaliação de EAs descritos na literatura ${ }^{4}$ - relatórios voluntários de incidentes, relatórios espontâ- 
neos com alerta, observação direta, revisão retrospectiva e prospectiva de prontuários, entrevista com pacientes, ou a combinação deles. A revisão retrospectiva de prontuários, método utilizado nessa pesquisa, foi o empregado nos principais estudos sobre ocorrência de EAs. Alguns autores apontam limitações na metodologia de revisão retrospectiva de prontuários. A primeira delas é a impraticabilidade para o uso rotineiro em serviços de saúde ${ }^{4}$. Outra limitação referese à dificuldade de identificação de EAs de menor gravidade ${ }^{18}$. Por último, tais autores apontam problemas na confiabilidade mais baixa entre os revisores não médicos, que atuam na fase de rastreamento ${ }^{18}$, e entre os revisores médicos. Essa metodologia foi empregada na maioria dos estudos que realizaram amplos diagnósticos sobre a ocorrência de EAs em hospitais e foram a base do desenvolvimento de estratégias de gestão da segurança dos pacientes em diversos países.

Este artigo apresenta o processo de adaptação dos instrumentos de avaliação de EA desenvolvidas pelo CAES para sua aplicação em pesquisa sobre a incidência de EA em hospitais brasileiros. A estratégia de adaptação adotada buscou compatibilizar os instrumentos de avaliação com a cultura médica e hospitalar brasileira, mantendo, no entanto, a comparabilidade dos resultados da pesquisa com aqueles obtidos em estudos internacionais.

\section{Metodologia}

A metodologia selecionada para avaliação de EA em hospitais brasileiros foi a mesma utilizada pelo CAES. A metodologia de revisão retrospectiva de prontuários baseiase numa fase inicial de avaliação, orientada por critérios explícitos de rastreamento de EA, seguida de uma fase de avaliação implícita estruturada para confirmação da ocorrência de EA.

Após solicitação, a equipe do CAES colocou à disposição deste estudo os seus instrumentos de avaliação. Esses estão contidos no Manual for the Canadian Adverse Events Study, composto por 5 documentos e 340 páginas: I) Operations Manual, que apresenta o panorama geral do estudo, o estudo de confiabilidade, as questões éticas e de confidencialidade do estudo e a equipe canadense de pesquisadores; II) Definitions and Protocol Section, que contém um dicionário com a definição da terminologia utilizada no estudo, seus objetivos, os critérios de seleção dos hospitais, o desenho da amostra pesquisada e os mecanismos de armazenamento e segurança dos dados; III) General Information Section, que apresenta as instruções para o preenchimento do formulário e as instruções de navegação no programa computacional; IV) Phase 1 Review, que contém o formulário de rastreamento (FR) e o glossário de termos a ser empregado na primeira etapa da avaliação de EA, baseada em critérios explícitos e realizada por enfermeiros; V) Phase 2 Review, que contém o formulário de avaliação de EA (FA) e o glossário de termos a ser empregado na etapa de avaliação propriamente dita, baseada em critérios implícitos e realizada por médicos.

No caso deste estudo, os formulários FR e FA e seus respectivos glossários e instruções de preenchimento foram informatizados. Todos os documentos de avaliação constam no Programa Computacional Eventos Adversos, criado para a pesquisa, não havendo qualquer material em papel. O desenvolvimento do programa computacional ocorreu ao longo de todo o processo de adaptação dos formulários (Figuras 1 e 2).

A adaptação dos instrumentos de avaliação foi realizada em 4 fases. A primeira fase consistiu na tradução inicial dos dois formulários (FR e FA), escritos originalmente em língua inglesa. Foram realizadas duas traduções da versão original para o português e posterior comparação entre elas, etapa em que se avaliou cada item traduzido quanto à clareza e adequação à linguagem e à realidade médica brasileira. $\mathrm{Na}$ segunda fase, realizou-se um painel de especialistas, formado por profissionais renomados em sua área de atuação, organizado para avaliar, com base em consen- 


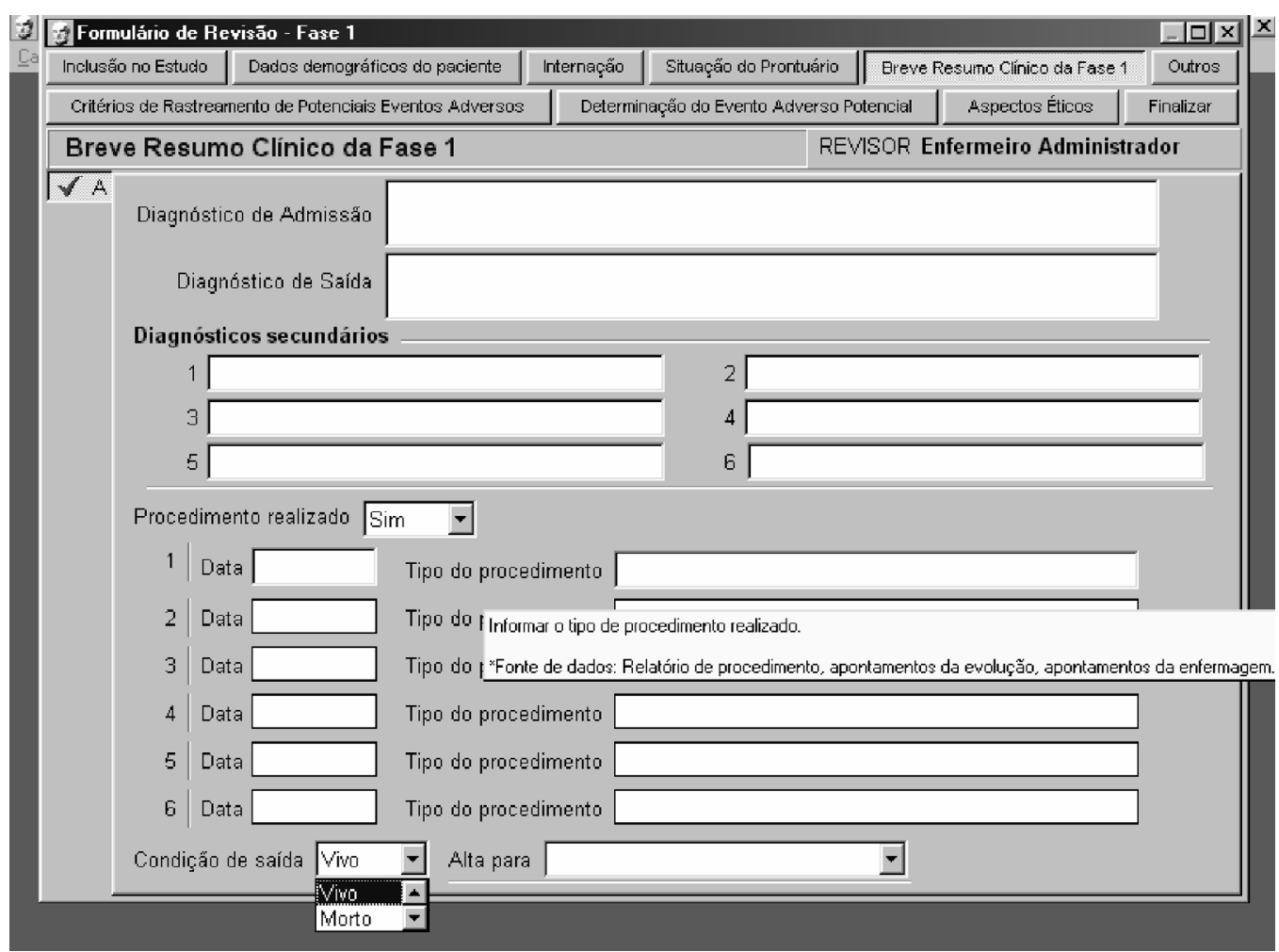

Figura 1 - Programa "Evento Adverso" - Formulário de Rastreamento (FR)

Figure 1 - "Adverse Event" Program - Phase 1 Form

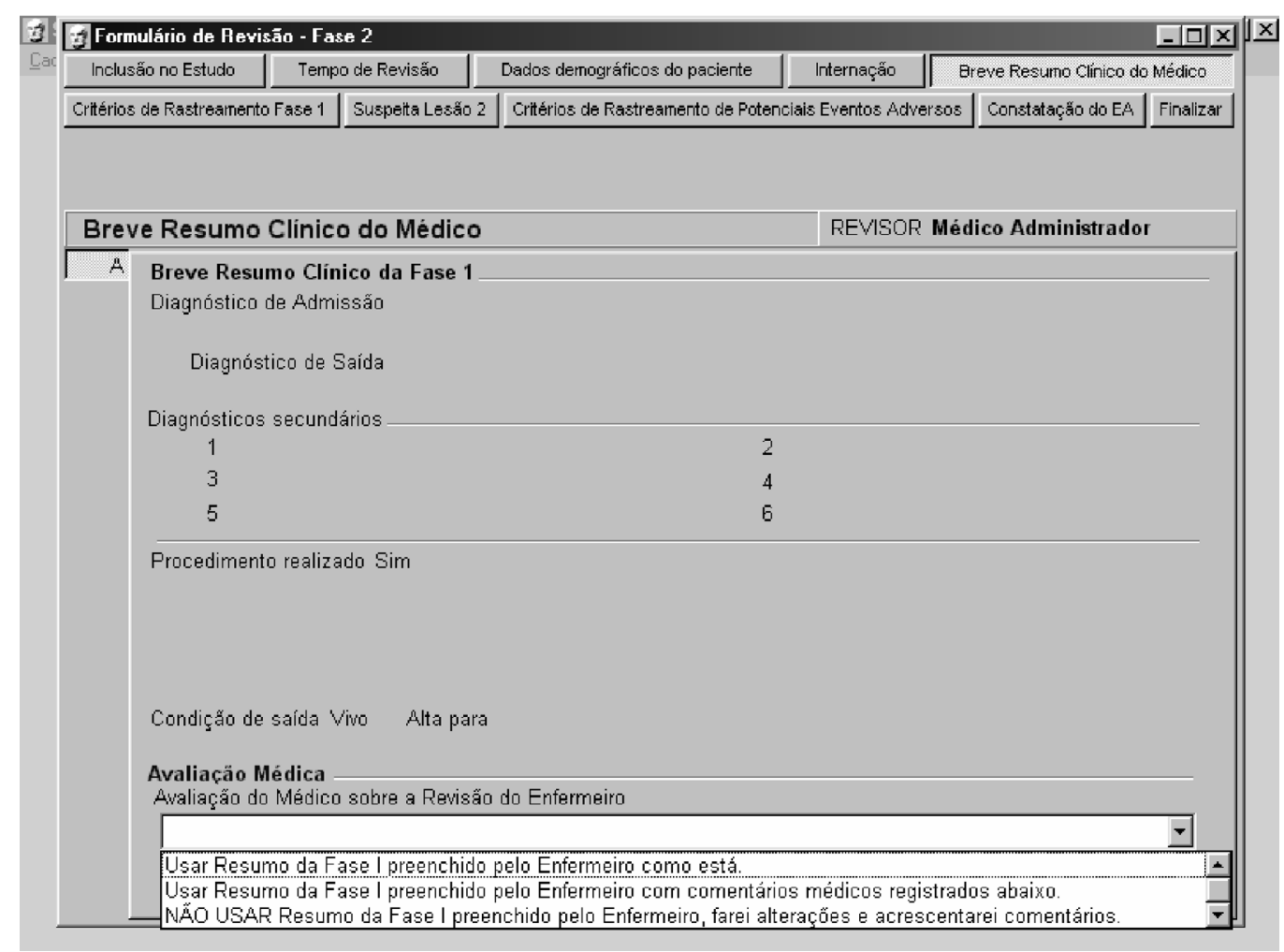

Figura 2 - Programa "Evento Adverso" - Formulário de Avaliação (FA)

Figure 2 - "Adverse Event" Program - Phase 2 Form 
so, os critérios de rastreamento a serem utilizados no estudo brasileiro e decidir sobre as discordâncias nas traduções dos formulários FR e FA. A terceira fase constituiu-se na realização de pré-teste para avaliar os prontuários, os revisores enfermeiros na etapa de rastreamento e o programa computacional. Por fim, na quarta fase, os formulários FR e FA adaptados foram retro-traduzidos [back translation] por um tradutor profissional, que realizou a retrotradução dos formulários sem o conhecimento da versão original. Esse processo mostrou-se necessário para a análise da equivalência do instrumento nos dois idiomas.

No processo inicial de tradução dos formulários FR e FA, foram realizadas duas traduções simultâneas, uma pela equipe de pesquisa e outra por tradutor profissional. Tal decisão foi tomada visando a comparação entre as duas versões para melhor adequação do instrumento à realidade brasileira. Nesta comparação foram verificadas algumas divergências e buscou-se, a partir daí, escolher que termo seria adotado como correspondente ao original em inglês e que mudanças seriam necessárias no formulário. Buscou-se obter equivalência semântica (equivalência entre as palavras quanto ao sentido que veiculam e à abrangência do mesmo), idiomática (substituição de itens e equivalência de expressões típicas, cujo significado não pode ser depreendido através de suas palavras individuais ou de seu sentido literal) e experimental (adequação de palavras ao contexto cultural alvo $)^{19}$. As dúvidas ou discordâncias sobre a melhor tradução foram encaminhadas para o painel de especialistas.

A segunda fase de adequação dos formulários voltou-se para a adaptação dos critérios de rastreamento de EAs. Os critérios de rastreamento (18 no CAES) são utilizados na fase inicial da avaliação para selecionar prontuários com potenciais EAs nos estudos de revisão retrospectiva de prontuários. Organizou-se o painel de especialistas, composto por 9 médicos de diversas especialidades (clínica médica, cardiologia, cirurgia geral, anestesia, infectologia, emergência, terapia intensiva, obstetrícia e saúde pública, com enfoque em medicamentos), selecionados dentre aqueles que desfrutavam de reconhecida competência na especialidade. No convite para participar do painel, os especialistas receberam as informações sobre o projeto, a metodologia a ser empregada e o tempo estimado de duração dos trabalhos. Os especialistas não foram remunerados. O método Delphi modificado ${ }^{20}$ foi empregado para obtenção de consenso no painel de especialistas. O objetivo desse método é conseguir uma opinião coletiva sobre determinadas questões, através de uma técnica de estruturação de um processo de comunicação colegiada, que permite a um grupo de indivíduos tratar de problemas complexos ${ }^{21}$.

Solicitou-se que os especialistas julgassem a pertinência da inclusão neste estudo de cada um dos 18 critérios de rastreamento de EA do CAES. Em 5 dos 18 critérios do CAES, também se apresentaram ao painel formulações alternativas empregadas nos estudos anteriores. Nesses casos, antes de julgar o critério, os especialistas escolhiam a formulação de sua preferência. Os especialistas responderam a três quesitos sobre cada critério: (I) se o critério de rastreamento de EA era um bom sinalizador para detectar evento adverso; (II) se era compatível com a realidade dos hospitais brasileiros; e (III) se estava formulado com linguagem clara e terminologia correta. Foi facultada ao especialista a sugestão de texto alternativo para cada critério. Cada quesito acima foi julgado a partir de uma escala de 1 a 5: 1 (definitivamente não); 2 (provavelmente não); 3 (provavelmente sim); 4 (muito provavelmente sim); e 5 (definitivamente sim). Quesitos com média maior que 4 foram considerados incluídos no estudo, entre 3 e 4 foram reavaliados pela equipe de pesquisa e menor que 3 foram excluídos. Entretanto, foram mantidos no estudo todos os critérios de rastreamento do CAES e incluídos aqueles sugeridos pelo painel de especialistas, de forma a permitir, na análise, comparabilidade ao estudo canadense. Os médicos especialistas também foram solicitados a julgar a melhor tradução 
de termos sobre os quais não houve consenso na equipe de pesquisa sobre a tradução mais adequada ou termos chave para o estudo.

Um pré-teste foi realizado para avaliar os prontuários, os revisores enfermeiros na etapa de rastreamento e o programa computacional. O pré-teste foi efetuado em prontuários de um hospital geral, público e de ensino da cidade do Rio do Janeiro, com a participação de duas duplas de revisores enfermeiros treinados. Cada dupla de revisores atuou em conjunto na avaliação dos prontuários. Foram analisados 175 prontuários de pacientes internados em 2003, selecionados aleatoriamente. Os pacientes com idade menor do que 18 anos, pacientes que permaneceram menos do que 24 horas no hospital e pacientes psiquiátricos foram excluídos. Analisou-se a concordância entre as duplas de enfermeiros em 29 prontuários a partir da concordância simples com intervalo de confiança de $95 \%$.

Na última fase, um novo tradutor profissional especializado retro-traduziu os formulários adaptados e essa tradução foi comparada aos textos originais dos formulários do CAES em inglês.

\section{Resultados}

Na tradução inicial dos formulários, a equipe de pesquisadores identificou discordâncias entre a sua tradução e aquela realizada pelo tradutor profissional. Como exemplos de discordância semântica entre as duas traduções, destacam-se o vocábulo discharge, que foi traduzido como "alta" pelo tradutor profissional e modificado para "saída" pelos pesquisadores, visando englobar a alta e o óbito, conforme norma do Ministério da Saúde; e o termo diagnostic radiology, considerado pelo tradutor como "diagnóstico radiológico”, mas substituído por "diagnóstico de imagem" por este ser mais abrangente e mais coerente com o propósito do termo no formulário canadense. Embora a tradução da expressão doctors orders pelo tra- dutor tenha acarretado divergência semântica em relação à tradução dos pesquisadores, optou-se pela tradução do profissional, "ordens médicas", em vez da tradução da equipe, "prescrições médicas”, que representa apenas uma entre as várias possibilidades de ordens médicas, termo de sentido mais abrangente.

Como exemplos de discordância idiomática tem-se: (I) para o termo index hospitalization, que indica a internação objeto da avaliação, a equipe de pesquisadores adotou o termo "internação índice" em vez de "internação em referência”, indicado pelo tradutor; (II) o termo medical progress notes, traduzido como "apontamentos médicos", foi modificado pelo termo indicado na tradução da equipe ("evolução médica”); (III) o termo admission history and physical, traduzido como "histórico da internação e exames clínicos”, foi traduzido pela equipe como "anamnese e exames físicos".

Houve casos em que a concisão da língua inglesa transformou-se em empecilho para a tradução de determinados vocábulos para o português, por esta língua não apresentar em seu léxico uma palavra que pudesse expressar sozinha o mesmo sentido do termo original. O caso mais relevante foi o vocábulo injury, traduzido como lesão ou dano, palavras sinônimas, mas de abrangência de significação e uso distintos na área médica. Nesse caso, o painel de especialistas entendeu que "lesão" não era suficiente para expressar corretamente a palavra injury, que passou a ser traduzida como "lesão ou dano". O mesmo se deu com a palavra disability, que passou a ser traduzida como "incapacidade ou deficiência”, e com os vocábulos impairment e harm, traduzidos como "dano". O termo wrong time (referindo-se à administração de medicamentos), traduzido como "horário errado" é entendido como o momento errado de administrar o medicamento. $\mathrm{O}$ termo inadequate handover foi traduzido como "passagem inadequada do caso".

As mudanças semânticas e idiomáticas realizadas estão intrinsecamente ligadas à 
obtenção da equivalência experimental, uma vez que o objetivo da comparação das traduções é a aplicabilidade do formulário, que deve ser claro, coerente, conciso e adequado ao contexto brasileiro.

A equivalência experimental foi obtida em quase todos os itens, destacando-se como divergência a substituição realizada pelos pesquisadores da tradução do termo family physicians office, de "consultório médico de família” por "consultório médico", no capítulo "Local de ocorrência do evento adverso e especialidade" no FA.

Na retro-tradução foi observada apenas uma discordância importante com relação aos formulários do CAES. O critério 6 (Quadro 1) “Transferência não planejada de ou para outro hospital de cuidados agudos" foi adaptado do original "Unplanned transfer to another acute care hospital”. Apesar de não estar expressa no texto original a transferência de outro hospital para o hospital índice, a equipe de pesquisa optou por esta adaptação porque, no formulário, é solicitado ao revisor que informe se o hospital índice recebeu ou transferiu o paciente.

Ao longo da tradução, a equipe de pesquisadores achou necessário introduzir algumas alterações no texto dos formulários. Foram modificados os campos pontuais do formulário original, transformando-os em perguntas diretas com a finalidade de explicitar algumas instruções sobre o seu preenchimento. Por exemplo, no campo Situação do Prontuário: (I) o termo “departamento de emergência” no FR foi substituído pela frase "Na internação índice, o paciente foi admitido pelo setor de emergência do hospital ou chegou transferido do setor de emergência de outro hospital?" para explicitar se o paciente esteve ou não em uma emergência na vigência da internação índice; e (II) o termo "permanência no hospital" foi substituído pela frase "O paciente permaneceu mais de 24 horas no hospital?", que explicita se o paciente ficou internado mais de 24 horas.

Alguns termos foram excluídos por não estarem adequados ao contexto cultural. Os serviços de parteira e de medicina da família foram suprimidos porque não existem serviços equivalentes em hospitais brasileiros. Por outro lado, outros itens foram incluídos. No critério de rastreamento, por exemplo, à "reação adversa ao medicamento" foi acrescentada a informação sobre o nome do medicamento causador da possível reação, campo que não estava previsto no FR do CAES.

Decidiu-se incluir no FR as seguintes variáveis sociais: ocupação do paciente, raça/cor de pele e escolaridade do paciente, com o objetivo de permitir avaliação de variação na incidência de EA em pacientes socialmente distintos.

Com base no painel de especialistas, a definição de EA foi traduzida como "lesão ou dano não intencional que tenha resultado em incapacidade ou disfunção temporária ou permanente e/ou internação prolongada em conseqüência do cuidado prestado", tal como indicada na introdução desse artigo.

O painel de especialistas julgou os critérios de rastreamento em duas etapas. $\mathrm{Na}$ primeira etapa, o painel avaliou os 18 critérios traduzidos e adaptados do CAES. Em cinco critérios, foram apresentados ao painel critérios alternativos selecionados pela equipe de pesquisa, oriundos de outros estudos: Harvard Practice Medical Study, 1984 (HMPS $^{9}$; Utah-Colorado Medical Practice Study, 1992 (UCMPS) ${ }^{10}$. Em apenas um caso o critério alternativo foi aprovado; os demais critérios foram aprovados da forma como foram traduzidos (Quadro 1). No caso do critério modificado, "óbito inesperado" foi alterado para “óbito” tal como no HMPS (1984), pois o painel considerou que esse julgamento só poderia ser feito pelos revisores médicos. Entretanto, a fim de se manter comparabilidade com o CAES, foi acrescentado um campo no FR cuja informação solicitada é se o óbito havia sido esperado ou inesperado.

Cinco critérios foram modificados com o objetivo de torná-los mais precisos. No critério 5, o termo "semi-intensivos" foi acrescentado para adequá-lo à realidade dos hospitais brasileiros, já que vários pos- 
Quadro 1 - Critérios de rastreamento traduzidos do CAES e resultados do painel de especialistas.

Chart 1 - Triggers for potential Adverse Events translated and results of expert panel

Critérios de rastreamento submetidos ao painel de especialistas.

1. Internação não planejada (incluindo reinternação) como resultado de qualquer cuidado de saúde prestado durante os 12 meses anteriores à internação índice

2. Internação não planejada em qualquer hospital durante os 12 meses subseqüentes à alta da internação índice

3. Ocorrência de lesão no paciente durante a internação (inclusive qualquer dano, lesão ou trauma ocorrido durante a internação índice)

4. Reação adversa ao medicamento

5. Transferência não planejada para unidade de cuidados intensivos

6. Transferência não planejada de ou para outro hospital de cuidados agudos, (excluindo as transferências para exames, procedimentos, ou cuidados especializados não disponíveis no hospital de origem)

7. Retorno não planejado à sala de cirurgia.Critério alternativo (UCMPS) - Procedimento cirúrgico "aberto" não planejado após laparoscopia ou admissão após um procedimento invasivo "fechado" ou laparoscópico planejado.

8. Remoção, lesão ou correção não planejada de um órgão ou estrutura durante cirurgia, procedimento invasivo ou parto vaginal. Critério alternativo (HMPS) - Tratamento de órgão lesado, após procedimento invasivo.

9. Outras complicações do paciente, p.ex. infarto agudo do miocárdio, AVC, embolia pulmonar etc. (incluir quaisquer complicações inesperadas) ocorridas durante a internação em referência que NÃO sejam um desenvolvimento normal da doença do paciente ou um resultado esperado do tratamento.

10. Desenvolvimento de déficit neurológico ausente na admissão, mas presente no momento da saída da internação índice (inclui déficits neurológicos relacionados aos procedimentos, tratamentos ou investigações).

\section{1. Óbito inesperadoCritério alternativo (HMPS) - Óbito}

12. Alta hospitalar inapropriada/ planejamento de alta inadequado para a internação índice (exclui alta à revelia).
Resultados do consenso dos especialistas.

Não aprovado

Não aprovado

Aprovado

Aprovado

Modificado - Transferência não planejada para unidade de cuidados intensivos e semiintensivos.

Modificado - Transferência não planejada de ou para outro hospital de cuidados agudos.

Aprovado o critério do CAES

Aprovado o critério do CAES

Modificado - Outras complicações inesperadas ocorridas durante a internação em referência que NÃO sejam um desenvolvimento normal da doença do paciente ou um resultado esperado do tratamento.

Modificado - Desenvolvimento de alteração neurológica ausente na admissão, mas no momento da saída da internação índice (incluem alterações neurológicas relacionados aos procedimentos, tratamentos ou investigações).

Aprovado critério alternativo (HMPS)

Aprovado. 
Quadro 1 - Continuação

Chart 1 - Continuation

Critérios de rastreamento submetidos ao painel de especialistas.

13. Parada cárdio-respiratória (com ressuscitação bem sucedida).
Resultados do consenso dos especialistas.

Modificado Parada cárdio-respiratória revertida.

Aprovado o critério do CAES

14. Lesão relacionada ao aborto ou trabalho de parto e partoCritério

alternativo (UCMPS) - Lesão ou complicação relacionada com

aborto, amniocentese, parto ou gravidez.

15. Infecção/septicemia hospitalar (excluir infecções/septicemia ocorridas em menos de 72 horas após a admissão).Critério alternativo (HMPS) - Temperatura corporal $>38.3^{\circ} \mathrm{C}$ no dia anterior à alta.

16. Insatisfação com o cuidado recebido, documentada no prontuário ou evidência de queixa apresentada (incluir documentos, queixas documentadas, conflitos entre o paciente/ família e profissionais e alta à revelia).

17. Documentação ou correspondência indicando litígio, seja somente intenção ou ação efetiva.

18. Partindo de uma creatinina normal na internação, houve duplicação do seu valor durante a permanência no hospital?

19. Quaisquer outros eventos indesejados não mencionados acima.
Aprovado o critério do CAES

Aprovado.

Aprovado.

Novo critério sugerido pelo painel de especialistas

Aprovado. suem unidades intermediárias às Unidades de Tratamento Intensivo (UTI) e às enfermarias. No critério 10, o termo "déficit neurológico” foi substituído por "alteração neurológica”. O critério 13, “parada cardíaca com ressuscitação bem sucedida”, foi modificado por "parada cardíaca revertida”, o que, no entendimento dos especialistas, corresponde ao termo equivalente no jargão médico brasileiro.

Os critérios 1 e 2 não foram aprovados pelo painel, que os considerou inadequados à realidade brasileira, pois ponderou não haver registro sistemático, nos prontuários dos pacientes, de detalhes de internações hospitalares anteriores e posteriores à internação índice.

Por fim, um novo critério foi incluído por sugestão dos especialistas: "Partindo de uma creatinina normal na internação, houve duplicação do seu valor durante a permanência no hospital?”. Este critério busca identificar os pacientes que desenvolveram, durante a internação, insuficiência pré-renal ou renal aguda, ou ainda agudização de insuficiência renal crônica.

No pré-teste, 175 prontuários foram analisados por uma dupla de enfermeiros e 29 desses foram posteriormente revistos por uma segunda dupla de enfermeiros treinada para avaliar a confiabilidade entre as duplas de revisores.

Entre os 175 prontuários analisados, $17 \%$ dos casos foram rastreados, isto é, selecionados pelos revisores enfermeiros como potenciais eventos adversos (pEAs). Essa proporção de pEAs foi bem menor do que a obtida pelo CAES (40,8\%). A concordância simples entre as duplas de revisores enfermeiros foi de $84 \%$ (IC $95 \%$ 69,9\% - 
98,4\%). Com base nesses resultados, foram levantadas duas hipóteses alternativas para explicar a baixa freqüência de pEAs: (I) prontuários com informação insuficiente; e (II) falhas dos revisores na etapa de rastreamento. A primeira hipótese indicaria a impossibilidade de aplicação do método de revisão retrospectiva de prontuários no hospital e a segunda indicaria falhas no treinamento dos revisores.

Para testar essas hipóteses, foram aleatoriamente selecionados 100 prontuários da mesma amostra, que foram avaliados por um médico membro da equipe da pesquisa e por 2 bolsistas graduandos de medicina. Nessa avaliação, a freqüência de pEA aumentou para 36,9\%, bem próxima da encontrada pelo CAES (40,8\%). Considerou-se que esse resultado reforçava a hipótese de falhas na avaliação e a necessidade de um novo treinamento dos revisores enfermeiros. Desse modo, optou-se por retreiná-los. $\mathrm{O}$ retreinamento foi baseado na metodologia de treinamento dos revisores aplicada no CAES.

No treinamento e na avaliação do aprendizado utilizaram-se prontuários previamente avaliados, aqui denominados de prontuários-padrão. Os prontuáriospadrão foram identificados em conjunto por 2 médicos clínicos, 2 acadêmicos de medicina e 1 médico do grupo da pesquisa, aplicando o FR e o FA. Foram selecionados 26 prontuários: 16 apresentavam um ou mais critérios de rastreamento positivos. Esses 16 prontuários correspondem aos casos com pEA, que teriam que ser selecionados pelos enfermeiros para posterior avaliação pelos médicos.

Na nova metodologia de treinamento, na primeira etapa apresenta-se a metodologia de avaliação e o FR. Em seguida, cada enfermeiro avalia em conjunto com um médicoinstrutor 5 prontuários-padrão. Na segunda etapa, os revisores avaliam isoladamente os 21 prontuários-padrão restantes. Na avaliação do treinamento, o avaliador deve atingir uma concordância de no mínimo $80 \%$ com os critérios de rastreamento nos prontuários-padrão. Todos os 4 enfermeiros obtive- ram concordância acima de $80 \%$ após treinamento.

\section{Discussão}

Aocorrência de EAs é considerada um problema de importância internacional. Observase recentemente uma intensificação de esforços na área da segurança do paciente por parte de agências nacionais e internacionais. Como conseqüência, há um envolvimento crescente de pesquisadores, profissionais de saúde e gestores de saúde com o desenvolvimento de avaliação de $\mathrm{EAs}^{4}$.

A OMS criou em 2002 um Grupo de Trabalho com o objetivo de estudar metodologias para avaliar de forma sistemática a segurança do paciente nos serviços de saúde, que resultou na criação, em 2005, da World Alliance for Patient Safety para desenho, planejamento e monitoramento de ações para a segurança do paciente, integradas entre os países membros ${ }^{22}$. Em setembro de 2007, a Comunidade Européia, em associação com a World Alliance for Patient Safety, realizou uma conferência na cidade do Porto, em Portugal, para definir uma agenda européia de pesquisa na área da segurança do paciente. Na atualidade, são inúmeras as instituições, redes de relacionamento e websites dirigidos para esse tema.

A experiência vivida por vários países desenvolvidos e, mais recentemente, por países em desenvolvimento na abordagem da segurança do paciente foi marcada por uma fase inicial de diagnóstico. O diagnóstico foi obtido a partir de estudos sobre a ocorrência deEAs nos serviços de saúde, que identificaram sua freqüência, natureza, principais causas e gravidade. Estudos com a abordagem apresentada neste artigo voltam-se fundamentalmente para a etapa diagnóstica.

Essa etapa cumpre o papel crucial de chamar atenção para o problema da ocorrência de erro nos serviços de saúde, além de produzir informações detalhadas sobre os EAs, de modo a orientar a definição de prioridades e o desenho de políticas e de intervenções nos serviços voltadas para tornar o cuidado à saúde mais seguro. 
No Brasil, não há ainda um diagnóstico amplo sobre os problemas de segurança do paciente em nossos hospitais. Os instrumentos apresentados neste artigo são base para estudos diagnósticos. Destacase que a aplicação desses instrumentos permite a comparação da incidência de EAs em hospitais no Brasil com aquela observada em outros estudos realizados no Canadá, Austrália, Nova Zelândia e Inglaterra, entre outros. A comparação entre estudos representa uma estratégia importante na avaliação do desempenho dos serviços e dos sistemas de saúde. A comparação do desempenho busca associar os resultados com as características dos sistemas e serviços de saúde, de modo a indicar o potencial de melhoria existente e as possíveis estratégias para sua obtenção.

Uma condição necessária para a comparação dos resultados de diferentes estudos é a aplicação de instrumentos de avaliação padronizados. Na adaptação do instrumento do CAES, o emprego de duas traduções para o português e as mudanças indicadas pelo painel de especialistas promoveram uma melhoria desses instrumentos no sentido de adequá-los à realidade brasileira. As mudanças necessárias foram realizadas, mantendo-se o sentido da comparabilidade dos resultados da avaliação dos EAs do estudo brasileiro com os demais estudos internacionais e, em particular, com o estudo canadense.

A taxa de pEA, na avaliação realizada por médico da equipe da pesquisa, foi próxima ao valor obtido no CAES $(40,8 \%)$ e superior a encontrada pelos revisores enfermeiros antes do retreinamento (18\%), o que mostrou que os prontuários dos hospitais estudados apresentavam as informações necessárias para o emprego do método de revisão retrospectiva de prontuários e indicou a aplicabilidade do FR adaptado em hospitais no Brasil.

A área de pesquisa sobre a segurança do paciente é relativamente recente e ainda existem questões importantes sobre a validade dos métodos empregados na mensuração de EAs. Entretanto, os métodos retrospectivos ${ }^{23}$ permanecem como os mais empregados na fase diagnóstica ou de mensuração de freqüência de EAs. Os instrumentos adaptados neste estudo podem ser empregados em associação com outros métodos na avaliação de EAs, além de servir de base para o desenvolvimento de instrumentos voltados para o monitoramento de intervenções de gestão da segurança do paciente.

\section{Referências}

1. Mendes W, Travassos C, Martins M, Noronha JC. Revisão dos estudos de avaliação da ocorrência de eventos adversos em hospitais. Rev Bras Epidemiol 2005; 8(4): 393-406.

2. Kohn LT, Corrigan JM, Donaldson MS, McKay T, Pike KC. To err is human. Washington, DC: National Academy Press; 2000.

3. Gallotti, RMD. Eventos adversos e óbitos hospitalares em serviço de emergências clínicas de um hospital universitário terciário: um olhar para a qualidade da atenção [tese de doutorado]. São Paulo: Faculdade de Medicina da Universidade de São Paulo; 2003.

4. Murff HJ, Patel VL, Hripcsak G, Bates DW. Detecting adverse events for patient safety research: a review of current methodologies. JAMIA 2003; 36:131-43.
5. Corrigan JM, Kohn LT, Donaldson MS, Maguire SK, Pike KC. Crossing the quality chasm: a new health system for the 21st century. Washington, DC: National Academy Press; 2001.

6. Studdert DM, Brennan TA, Thomas EJ. What have we learned since the Harvard Medical Practice Study? In: Rosenthal MM, Sutcliffe, Kathleen M (eds.). Medical Error: what do we know? What do we do?. EUA: JosseyBass; 2002. p. 3-34.

7. World Health Organization. Patient Safety: Rapid Assessment Methods for Estimating Hazards. Genebra; 2003.

8. Mills DH. Medical insurance feasibility study. A technical summary. West J Med 1978; 128: 360-5. 
9. Lilford R, Mohammed M, Spiegelhalter D, Thomson R. Use and misuse of process and outcome data in managing performance of acute medical care: avoiding institutional stigma. Lancet 2004; 363: 1147-54.

10. Studdert DM, Brennan TA. No-fault compensation for medical injuries: the prospect for error prevention. JAm Med Assoc 2001; 286:217-23.

11. Wilson RM, Runciman WB, Gibbert RW, Harrison BT, Newby L, Hamilton JD. The Quality in Australian Health Care Study. Med J Aust 1995; 163: 458-71.

12. Davis P, Lay-Yee R, Schug S, Briant R, Scott A, Johnson S et al. Adverse events regional feasibility study: indicative findings. NZMed J2001; 114: 203- 5.

13. Vincent C, Neale G, Woloshynowych M. Adverse events in British hospitals: preliminary retrospective record review. Br Med J2001; 322: 5179

14. Michel P, Quenon JL, Sarasqueta AM, Scemama O. Comparison of three methods for estimating rates of adverse events and rates of preventable adverse events in acute care hospitals. Br Med J 2004; 328: 199-202.

15. Schioler T, Lipczak H, Pedersen BL, Mogensen TS, Bech $\mathrm{KB}$, Stockmarr A et al. Danish Adverse Event Study, Incidence of adverse events in hospitals. A retrospective study of medical records. Ugeskr Laeger 2002; 164: 43779 .

16. Baker GR, Norton PG, Flintoft V, Blais R, Brown A, Cox J et al. The Canadian Adverse Events Study: the incidence of adverse events among hospital patients in Canada. Can Med Assoc J2004; 170: 1678-86.
17. Aranaz JM, Estudio Nacional Sobre Los Efectos Adversos Ligados a La Hospitalización - ENEAS 2005. Informe febrero 2006, Ministerio de Sanidad y Consumo.

18. Lilford R, Mohammed M, Spiegelhalter D, Thomson R. Use and misuse of process and outcome data in managing performance of acute medical care: avoiding institutional stigma. Lancet. 2004; 363: 1147-54

19. Duarte, PS, Miyazaki, OS, Ciconelli, RM, Sesso Tradução e adaptação cultural do instrumento de avaliação de qualidade de vida para pacientes renais crônicos (KDQOL-SF). Rev Assoc Med Bras, 2003; 49(4): 375-81.

20. Fitch K, Berstein SJ, Aguilar MD, Burnand B, LaCalle JR, Lázaro P et al. The RAND/UCLA Appropriateness Method User's Manual. RAND; 2001.

21. Piola SF, Vianna SM, Consuelo DV. Estudo Delphi: atores sociais e tendências do sistema de saúde brasileiro. Cad Saúde Pública 2002; 18(Supl): 181-90.

22. World Health Organization.World. Alliance for Patient Safety: forward programme. Genebra; 2005.

23. Michel, P. Strengths and Weaknesses of Available Methods for Assessing the Nature and Scale of Harm Caused by the Health System: Literature Review. World Health Organization; 2003.

Recebido em: 03/01/07

Versão final reapresentada em: 16/10/07

Aprovado em: 04/12/07 